\title{
Recent progress at the Palomar Testbed Interferometer
}

\author{
R. L. Akeson
}

R. L. Akeson, "Recent progress at the Palomar Testbed Interferometer," Proc. SPIE 6268, Advances in Stellar Interferometry, 62680E (27 June 2006); doi: 10.1117/12.670571

Event: SPIE Astronomical Telescopes + Instrumentation, 2006, Orlando, Florida, United States 


\title{
Recent Progress at the Palomar Testbed Interferometer
}

\author{
R. L. Akeson ${ }^{\mathrm{a}}$, and the PTI Collaboration \\ ${ }^{a}$ Michelson Science Center, Caltech 100-22, Pasadena, CA, USA 91125
}

\begin{abstract}
The Palomar Testbed Interferometer (PTI) is a long-baseline, near-infrared interferometer located on Palomar Mountain, California. PTI has been conducting science operations since 1997 and continues to function both as a productive scientific instrument and as a technical testbed. Here I will review the current status and near-term plans for PTI, highlight some of the science results of the last two years, and describe the recently released PTI data archive.
\end{abstract}

Keywords: long-baseline interferometry, data archive

\section{INTRODUCTION}

The Palomar Testbed Interferometer (PTI) is a long-baseline, fringe-tracking interferometer which operates in the nearinfrared bands ( $\mathrm{H}$ and K-band) and is located at the Palomar Observatory, Palomar Mountain, California. PTI was constructed by the Jet Propulsion Laboratory, Caltech, with funding from the National Aeronautics and Space Administration as a technical testbed for the Keck Interferometer, specifically for demonstration of dual-star, narrowangle astrometry and phase referencing between two adjacent stars. PTI obtained first fringes in 1995 and has been in routine science operations since 1997. PTI science contributions previous to 2004 were reviewed by Boden ${ }^{1}$ at the previous meeting of this conference and this paper will concentrate on results since then.

A full technical description of PTI can be found in Colavita et $\mathrm{al}^{2}$. PTI currently has three operational stations, which can be combined pair-wise into three baselines, two $\sim 85$ meters and one $\sim 110$ meters in length, resulting in typical fringe spacings of 4 to 5 milliarcsecs at K-band (2.2 microns). PTI has a high science data throughput due to the efficient fringe tracking and the highly automated nighttime operations which are run in a queue-scheduled mode.

\subsection{Current Operations}

PTI currently operates regularly in two modes, visibility amplitude and fringe scanning. The fringe scanning mode is described by Mutterspaugh et al. ${ }^{3}$ and is used by the PHASES (Palomar High-precision AStrometric Exoplanet Search S. Kulkarni, PI) Key Project. The visibility amplitude observations encompass a range of smaller programs and one Key Science project, which uses PTI to survey candidate Terrestrial Planet Finder (TPF) target stars for companions (A. Boden, PI).

PTI is supported by the PTI collaboration (http://msc.caltech.edu/missions/Palomar) and administered by the Michelson Science Center (MSC) for the collaboration and the JPL/Caltech community. Funding for continued operations is provided by contributions from the MSC, the Space Interferometry Mission (SIM-PlanetQuest), the PHASES project, and the Infrared Processing and Analysis Center (IPAC). More information on available PTI support from the MSC can be found at http://msc.caltech.edu/software/PTISupport.

\subsection{Near-term plans}

PTI operations are fully funded through 2006 and operations are planned for 2007. The funding available for upgrades is limited, but some work is being pursued. M. Ireland is testing adding polarimetry to PTI and a design study has been done to implement the target tracking with infrared instead of visible light, which would enable observations of objects with red colors too faint in the visible for the current tracking system. 


\section{RECENT SCIENCE RESULTS}

A few of the recent science results with PTI are briefly described here. Please refer to the original reference for more information.

\subsection{Young stellar objects}

Eisner et al. ${ }^{4}$ observed 14 Herbig Ae/Be (HAEBE) sources in a continuation of the observations described in Eisner et $\mathrm{al}^{5}$. HAEBE stars are young A and B spectral type stars which are often surrounded by circumstellar gas and dust. All except two sources were resolved at $\mathrm{K}$ band ( 2.2 microns), with angular sizes generally $<\sim 5$ milliarcsec. They estimated the size scales and orientations of the 2.2 micron emission using various models: uniform disks, Gaussians, uniform rings, flat accretion disks with inner holes, and flared disks with puffed-up inner rims. Although it was not always possible to distinguish different radial distributions, the data were sufficient to place firm constraints on the inclinations of most sources; seven objects display significantly inclined morphologies. This is in contrast to previous work ${ }^{6}$ on HAEBE sources at shorter baselines which did not reveal significant inclinations. The derived inner disk inclinations are generally compatible with the outer disk geometries inferred from millimeter interferometric observations, implying that HAEBE disks are not significantly warped. Using the derived inner disk sizes and inclinations, Eisner et al. ${ }^{4}$ compute the spectral energy distributions (SEDs) for two simple physical disk models and compared these with observed SEDs compiled from the literature and new near-IR photometry. While geometrically flat accretion disk models were consistent with the data for the early B spectral types (consistent with previous work ${ }^{7}$ ), the later spectral type sources are explained better through models incorporating puffed-up inner disk walls. The different inner disk geometries may indicate different accretion mechanisms for early- and late-type HAEBE stars.

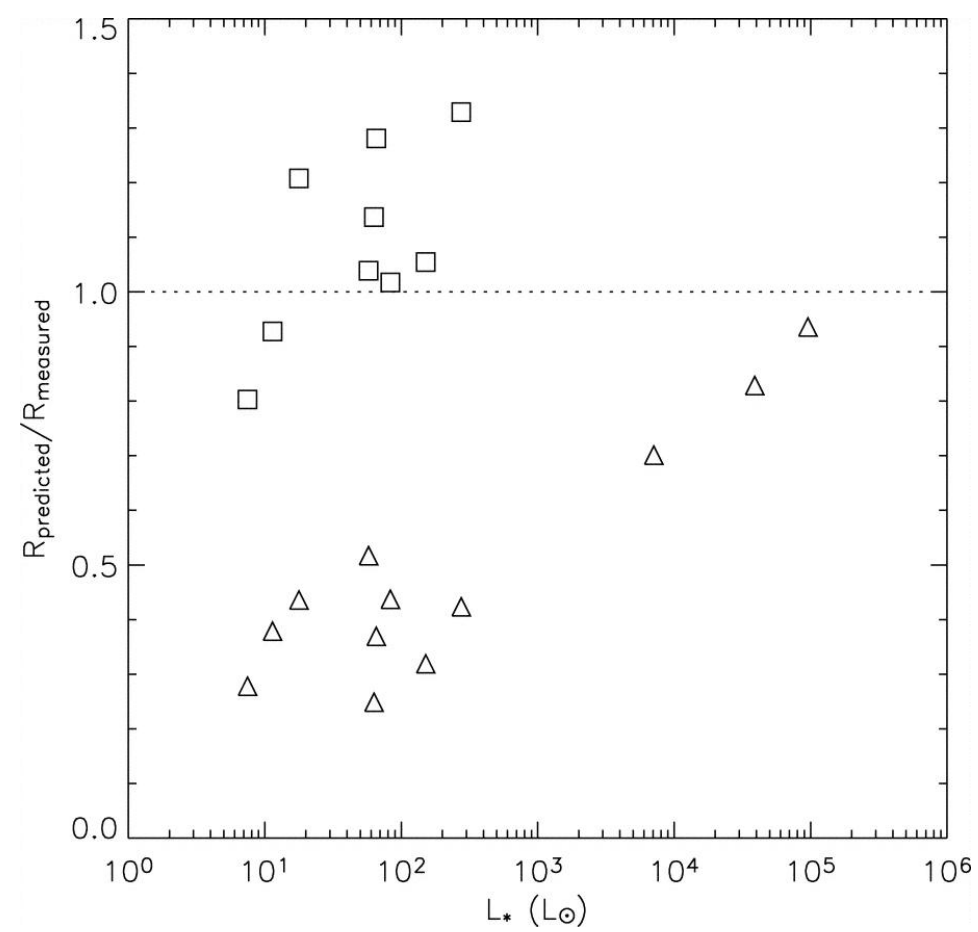

Figure 1: Ratio of measured to predicted inner disk sizes, as a function of stellar luminosity. The measured sizes are determined from near-IR interferometry for the geometrically flat-disk model (triangles) and the puffed-up inner rim model (squares). Figure taken from Eisner et al. ${ }^{4}$

Akeson et al. ${ }^{8}$ presented observations of four T Tauri stars (solar-mass young stars). The target sources, T Tau N, SU Aur, RY Tau, and DR Tau, are all known to be surrounded by dusty circumstellar disks. The observations directly trace 
the inner regions , < 1 Astronomical Unit (AU), of the disk and were used to constrain the physical properties of the disk material. For three of the sources observed, the infrared emission was clearly resolved. Geometric models were used to characterize the emission region size, which ranged from 0.04 to $0.3 \mathrm{AU}$ in radius. Monte Carlo radiation transfer models were then used to jointly model the spectral energy distribution and the interferometric observations with disk models including accretion and scattering. With these models, Akeson et $\mathrm{al}^{8}$ were able to reproduce the data set with extended emission arising from structures larger than 10 milliarcsec $(1.4 \mathrm{AU})$ contributing less than $6 \%$ of the K-band emission, consistent with little or no dusty envelope remaining for these sources. The radiation transfer models have inner radii for the dust similar to the geometric models; however, for RY Tau, emission from gas within the inner dust radius contributes significantly to the model flux and visibility at infrared wavelengths. The main conclusion from the Monte Carlo modeling is that emission from inner gas disks (between the magnetic truncation radius and the dust destruction radius) can be a significant component in the inner disk flux for sources with large inner dust radii.

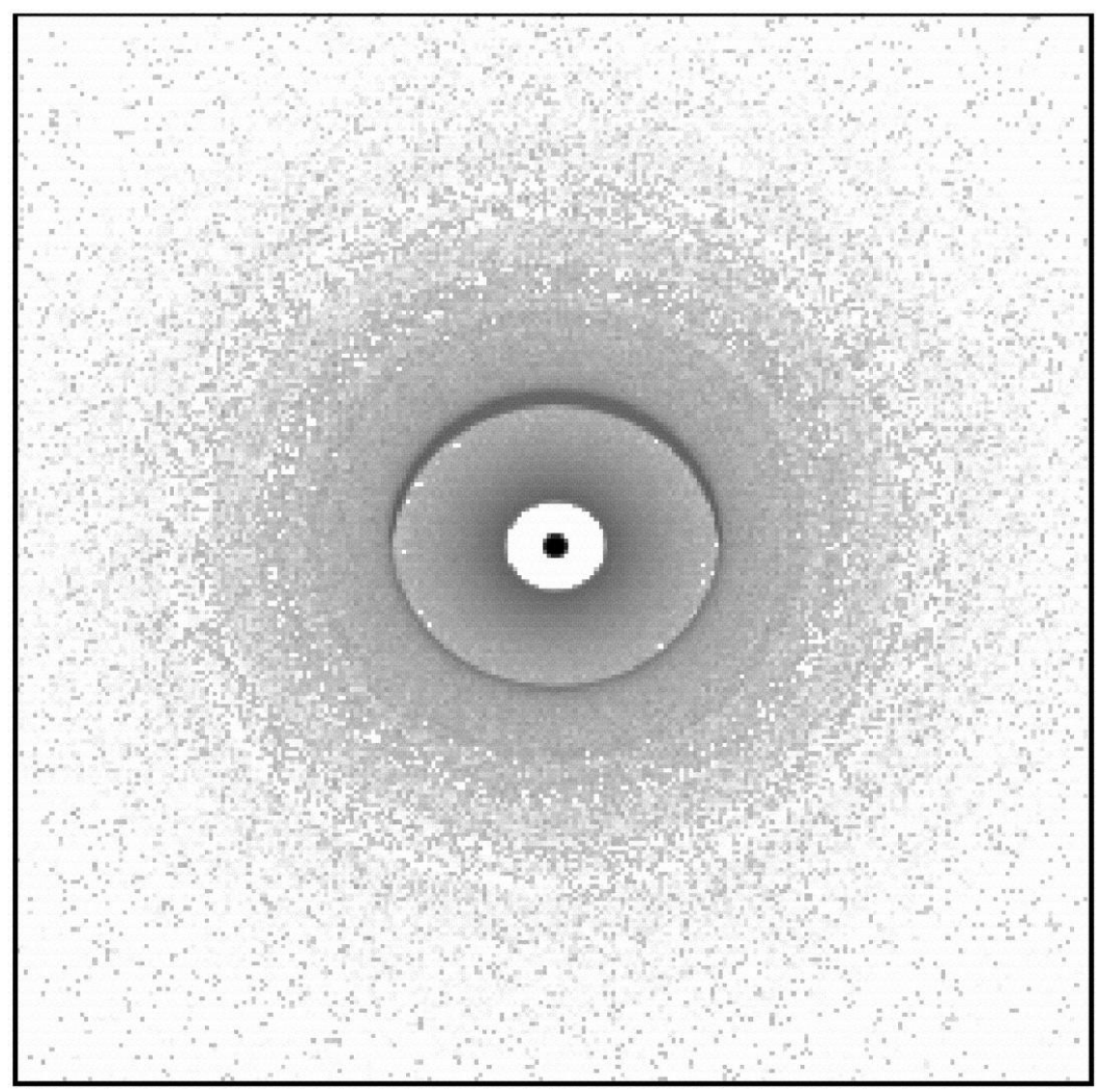

Figure 2. Monte Carlo model image for RY Tau at $\mathrm{K}$ band from Akeson et al. ${ }^{8}$ The flux has been scaled to the 0.15 power to provide better contrast in the image. The image is 12.5 mas or $1.75 \mathrm{AU}$ across. The outermost inclined structure is the inner edge of the dust disk and emission from gas can clearly be seen within this radius. The inclination angle shown represents the best fit value, but the position angle has been normalized.

\subsection{Binary stars}

12 Boo: Boden et al. ${ }^{9}$ reported a follow-on study of the double-line spectroscopic binary system 12 Bootis, in which the primary component is in a rapid phase of evolution, making an accurate measurement of its physical parameters an interesting test case for stellar evolution models. The 12 Boo interferometry data set includes 6 years with the Palomar Testbed Interferometer, a smaller amount of data from the Navy Prototype Optical Interferometer, and a radial velocity data set spanning 14 years from the Harvard-Smithsonian Center for Astrophysics. Boden et al. ${ }^{9}$ updated the 12 Boo physical orbit model with these expanded interferometric and radial velocity data sets, and the revised orbit is in good agreement with previous results ${ }^{10}$ from PTI. The physical parameters implied by a combined fit to the visibility and 
radial velocity data result in precise component masses and luminosities. In particular, the orbital parallax of the system is determined to be $27.72 \pm 0.15$ milliarcsec, and masses of the two components are determined to be $1.4160 \pm 0.0049$ and $1.3740 \pm 0.0045 \mathrm{M}_{\odot}$, respectively. These mass determinations are more precise than those in the previous report ${ }^{10}$ by a factor of 4-5. Although the two components are nearly equal in mass, the system exhibits a significant brightness difference in the near-infrared and visible. This brightness difference is attributed to evolutionary differences between the two components in their transition between main-sequence and giant evolutionary phases, and based on theoretical models, a system age of approximately $3.2 \mathrm{Gyr}$ is estimated. Comparisons with stellar models suggest that the 12 Boo primary may be just entering the Hertzsprung gap, but that conclusion is highly dependent on details of the models.

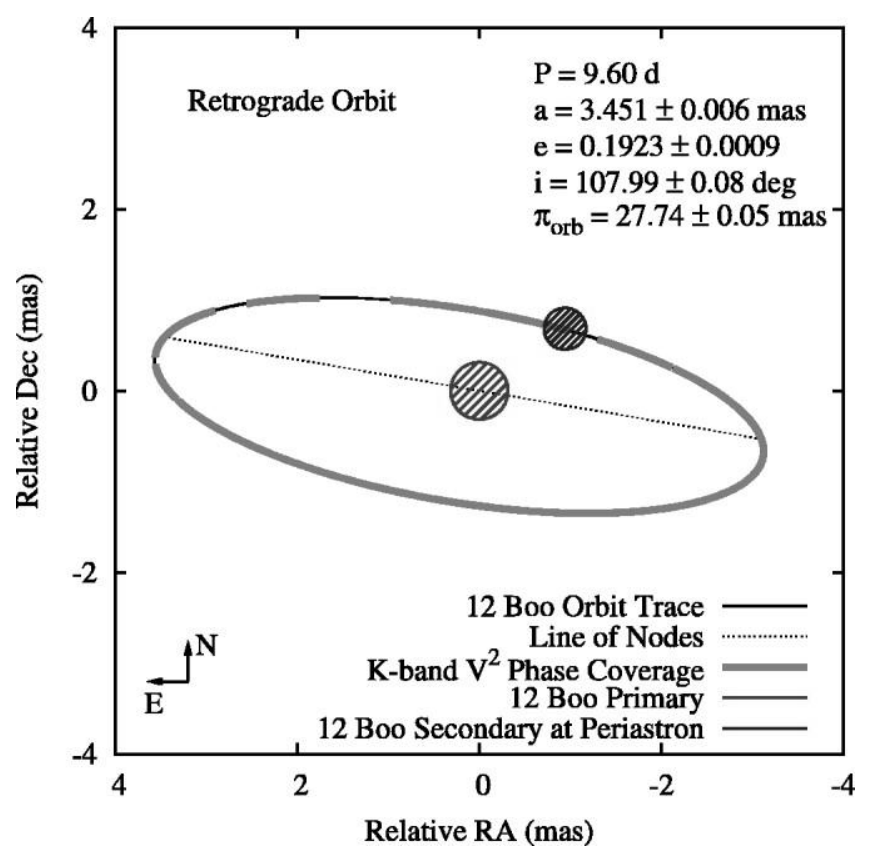

Figure 3: Figure taken from Boden et al. ${ }^{9}$ The relative visual orbit model of 12 Boo is shown with the primary and secondary objects rendered at $T_{0}$ (periastron). The heavy lines along the relative orbit indicate phase coverage in the $K$-band PTI data, which includes essentially all phases of the orbit. Component diameter values are estimated and are rendered to scale.

HD9939: Boden et al. ${ }^{12}$ reported on spectroscopic and interferometric observations of the high-proper motion doublelined binary system HD 9939, which has an orbital period of approximately 25 days. By combining radial-velocity and visibility measurements they estimated the system physical orbit and derived dynamical masses for the components of $\mathrm{M}_{\mathrm{A}}=1.072 \pm 0.014 \mathrm{M}_{\odot}$ and $\mathrm{M}_{\mathrm{B}}=0.8383 \pm 0.0081 \mathrm{M} \odot$; fractional errors of $1.3 \%$ and $1.0 \%$, respectively. A system distance of $42.23 \pm 0.21 \mathrm{pc}$ was determined, which corresponds to an orbital parallax of $\pi_{\mathrm{orb}}=23.68 \pm 0.12$ milliarcsec. The system distance and the estimated brightness difference between the stars in $\mathrm{V}, \mathrm{H}$, and $\mathrm{K}$ bands yielded component absolute magnitudes in these bands. By spectroscopic analysis and spectral energy distribution modeling they estimated the component effective temperatures and luminosities as $\mathrm{T}_{\text {eff }}(\mathrm{A})=5050 \pm 100 \mathrm{~K}, \mathrm{~T}_{\text {eff }}(\mathrm{B})=4950 \pm 200 \mathrm{~K}, \mathrm{~L}_{\mathrm{A}}=2.451 \pm$ $0.041 \mathrm{~L} \odot_{\odot}$, and $\mathrm{L}_{\mathrm{B}}=0.424 \pm 0.023 \mathrm{~L} \odot$. Both the spectral analysis and comparison with stellar models suggest that HD 9939 has elemental abundances near solar values. Further, comparison with stellar models suggests the HD 9939 primary has evolved off the main sequence and appears to be traversing the Hertzsprung gap as it approaches the red giant phase of its evolution. These measurements of the primary properties provide new empirical constraints on stellar models during this particularly dynamic evolutionary phase. As HD 9939 is currently in a relatively short-lived evolutionary state, the system age can be estimated as $9.12 \pm 0.25$ Gyr. In turn, the age and abundance of the system place a potentially interesting constraint on star formation in the galactic disk. 


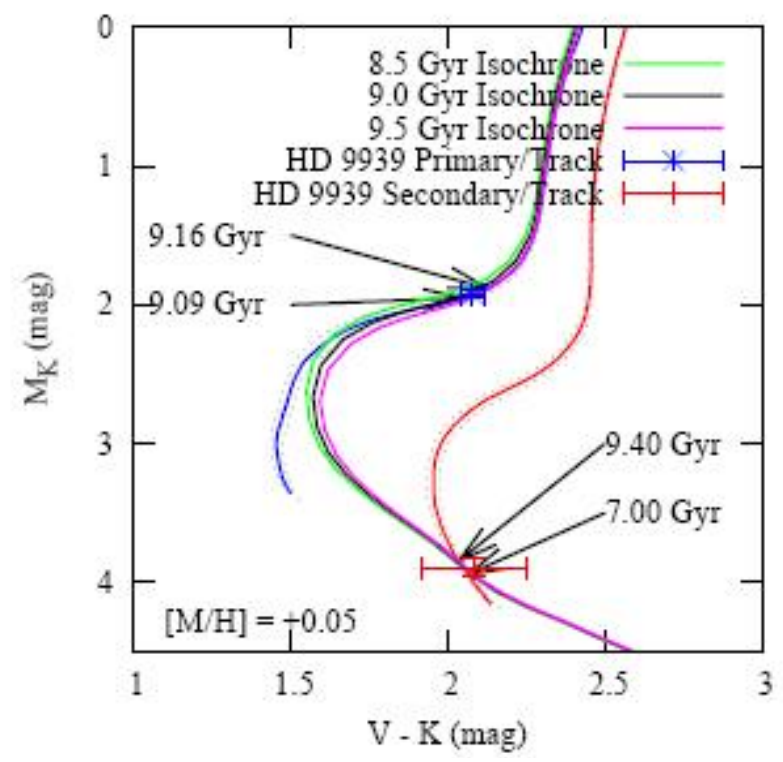

Figure 4: Detailed component model comparisons for HD 9939 from Boden et al. ${ }^{12}$ Best-fit evolutionary tracks and isochrones are shown for a metallicity of $[\mathrm{M} / \mathrm{H}]=0.05$.

\subsection{Novae and variable stars}

V838 Mon: Lane et al. ${ }^{12}$ used PTI to observe the peculiar outbursting variable V838 Mon, which is the second published observation of a nova-like outburst using an optical/near-IR interferometer-the only previous result ${ }^{13}$ being the observations of Nova Cyg 1992, made with the MkIII interferometer. The high angular resolution provided by PTI allowed Lane et al. ${ }^{12}$ to draw two preliminary conclusions. First, the source is resolved and has a characteristic angular size of a few milliarcseconds. Second, the difference in measured fringe visibility between the two baselines used is inconsistent with the source being a simple uniform disk. They explored other possible source morphologies, including inclined disks and binary models, and find that the data can be reasonably matched by such models, but further data are needed to constrain these models. Any model proposed to explain the outburst of V838 Mon must account for the presence of very extended, cool, asymmetric emission.

\section{PTI DATA ARCHIVE}

The MSC has developed an archive for the instrument-calibrated, averaged visibility data from the visibility amplitude mode. Access to the archive is through a web interface (https://mscweb.ipac.caltech.edu/mscdat-pti). Public users may self-register for an account. The web interface allows searching the data by source name, sky position, date, and baseline. The data can be downloaded in either ASCII or FITS formats. The MSC also provides a processing tool to calibrate this data for the system visibility; this tool is available for download or through a web interface. For more information, see http://msc.caltech.edu/software/PTISupport/.

In order to create a database useful to the entire astronomical community, a set of minimum criteria was established for inclusion in the database. Data which did not meet these criteria are still preserved at the MSC, but are not available in 
the on-line database. The criteria include passing a set of data validation and completeness checks. To increase the usefulness of the database, all source names have been converted to SIMBAD-compliant designations, except that internal spaces have been eliminated (i.e. HBC 123 is HBC123 in the PTI database). Within the database, sources are tagged as either targets or calibrators, allowing users to download all data necessary for the final calibration step. A catalog of PTI metadata will be available for searching through the NASA/IPAC Infrared Science Archive. The MSC is investigating extending the automatic processing and the database to include system-visibility calibrated data which can be used directly in astrophysical modeling.

The archive currently contains data from 1765 sources over 1061 nights for a total of over 300,000 measurements. As of March 2006, 706 nights are available to the public. The PTI sensitivity limits are roughly $\mathrm{V}<11$ and $\mathrm{K}<6$ with approximate declination limits of -10 to 60 degrees. Science targets observed with PTI cover a number of galactic astrophysical topics, including (but not limited to) binary orbits, radii of main sequence, giant and supergiant stars, and circumstellar material around evolved and young stars. The luminosity class, spectral type and SIMBAD identification (for those objects with designations other than Star, Variable star (unclassified), or High proper motion star) are shown in Figure 5, for the publicly available sources in the database.

This work was performed at the Michelson Science Center, Caltech and made use of services produced at the Michelson Science Center and the SIMBAD database, operated at CDS, Strasbourg, France.

\section{REFERENCES}

1. Boden, A., 2004, "Science and instrumentation update from the Palomar testbed interferometer", SPIE Proceedings 5491, 506.

2. Colavita, M. M., et al. 1999, "The Palomar Testbed Interferometer", Astrophysical Journal, 510, 505

3. Mutterspaugh, M., et al., 2006, "Scientific results from high-precision astrometry at the Palomar Testbed Interferometer", SPIE Proceedings, this volume

4. Eisner, J. A., Lane, B. F., Hillenbrand, L. A., Akeson, R. L., Sargent, A. I., 2004, "Resolved Inner Disks around Herbig Ae/Be Stars", Astrophysical Journal, 613, 1049

5. Eisner, J. A., Lane, B. F., Akeson, R. L., Hillenbrand, L. A., Sargent, A. I., 2003, "Near-Infrared Interferometric Measurements of Herbig Ae/Be Stars", Astrophysical Journal, 588, 360

6. Millan-Gabet, R., Schloerb, F. P., Traub, W. A., 2001, "Spatially Resolved Circumstellar Structure of Herbig AE/BE Stars in the Near-Infrared", Astrophysical Journal, 546, 358

7. Monnier, J. D., Millan-Gabet, R., 2002, "On the Interferometric Sizes of Young Stellar Objects", Astrophysical Journal, 579, 694

8. Akeson, R. L., Walker, C. H., Wood, K., Eisner, J. A., Scire, E., Penprase, B., Ciardi, D. R., van Belle, G. T., Whitney, B., Bjorkman, J. E., 2005, "Observations and Modeling of the Inner Disk Region of T Tauri Stars", Astrophysical Journal, 622, 440

9. Boden, A. F., Torres, G., Hummel, C. A., 2005, "Testing Stellar Models with an Improved Physical Orbit for 12 Bootis", Astrophysical Journal, 627, 464

10. Boden, A. F., Creech-Eakman, M. J., Queloz, D., 2000, "The Visual Orbit and Evolutionary State of 12 Bootis", Astrophysical Journal, 536, 880

11. Boden, A.F., Torres, G., Latham, D., 2006, "A Physical Orbit for the High Proper Motion Binary HD 9939", Astrophysical Journal, in press

12. Lane, B. F., Retter, A., Thompson, R. R., Eisner, J. A., 2005, "Interferometric Observations of V838 Monocerotis", Astrophysical Journal, 622, 137

13. Quirrenbach, A., Elias, N. M., II, Mozurkewich, D., Armstrong, J. T., Buscher, D. F., Hummel, C. A., 1993, "Observations of Nova Cygni 1992 with a long-baseline optical interferometer", Astronomical Journal, 106, 1118 

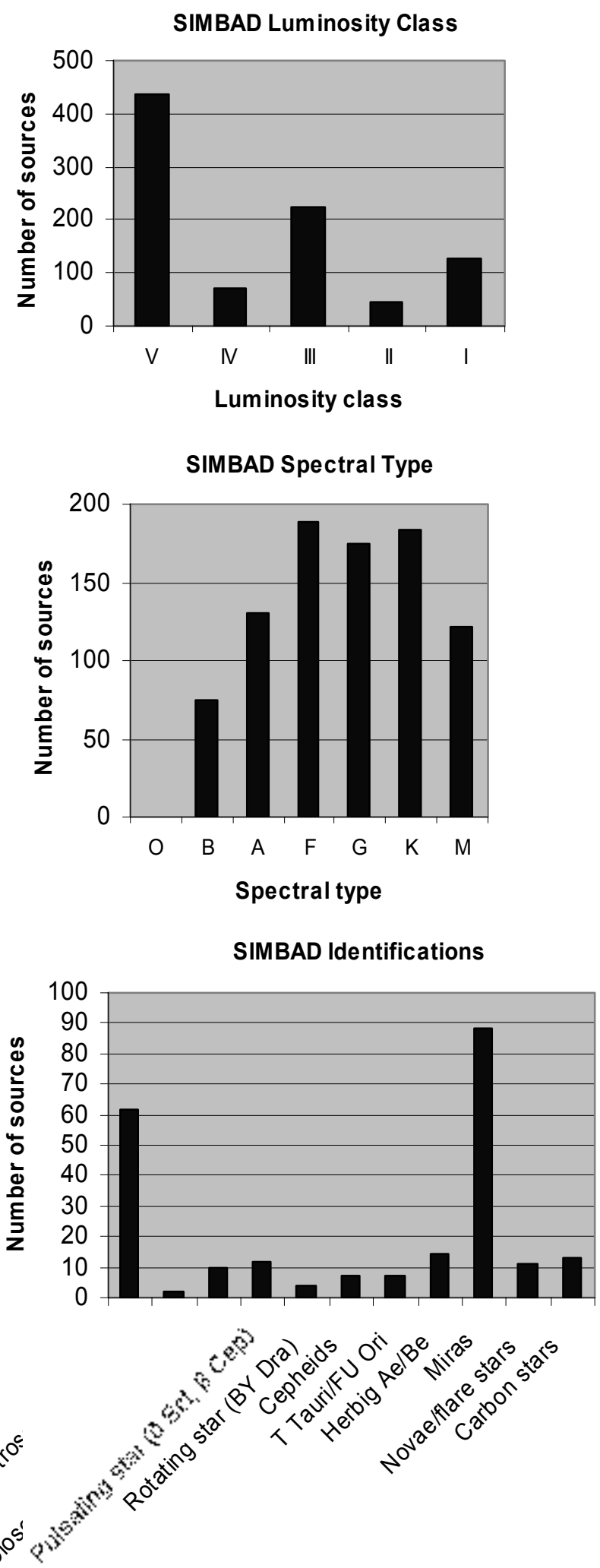

Figure 5: Luminosity class, spectral type and SIMBAD identification for public data in the PTI archive. Identifications are given only for stars with classifications other than Star, High proper motion star or variable star (unclassified). 